Pacific Journal of Mathematic 


\section{THE HANF NUMBER OF OMITTING COMPLETE TYPES}

\section{SAHARON SHELAH}

It is proved in this paper that the Hanf number $m^{C}$ of omitting complete types by models of complete countable theories is the same as that of omitting not necessarily complete type by models of a countable theory.

Introduction. Morley [3] proved that if $L$ is a countable firstorder language, $T$ a theory in $L, p$ is a type in $L$, and $T$ has models omitting $p$ in every cardinality $\lambda<\beth_{\omega_{1}}$, then $T$ has models omitting $p$ in every infinite cardinality. He also proved that the bound $\beth_{\omega_{1}}$ cannot be improved, in other words the Hanf number is $\beth_{\omega_{1}}$. $\mathrm{He}$ asked what is the Hanf number $m^{c}$ when we restrict ourselves to complete $T$ and $p$. Clearly $m^{C} \leqq \beth_{\omega_{1}}$. Independently several people noticed that $m^{C} \geqq \beth_{\omega}$ and J. Knight noticed that $m^{C}>\beth_{\omega}$.

Malitz [2] proved that the Hanf number for complete $L_{\infty, \omega}$ theories with one axiom $\psi \in L_{\omega_{1}, \omega}$ is $\beth_{\omega_{1}}$. We shall prove

\section{THEoREM 1. $m^{C}=\beth_{\omega_{1}}$.}

Notation. Natural numbers will be $i, j, k, l, m, n$, ordinals $\alpha, \beta, \delta$; cardinals $\lambda, \mu_{.}|A|$ is the cardinality of $A, \beth_{\alpha}=\sum_{\beta<\alpha} 2^{\beth_{\beta}}+\aleph_{0}$.

$M$ will be a model with universe $|M|$, with corresponding countable first-order language $L(M)$. For a predicate $R \in L(M)$, the corresponding relation is $R^{M}$ or $R(M)$, and if there is no danger of confusion just $R$. Every $M$ will have the one place predicate $P$ and individual constants $c_{n}$ such that $P=P^{M}=\left\{c_{n}: n<\omega\right\}, n \neq m \Rightarrow c_{n} \neq c_{m}$ (we shall not distinguish between the individual constants and their interpretation). A type $p$ in $L$ is a set of formulas $\varphi\left(x_{0}\right) \in L ; p$ is complete for $T$ in $L$ if it is consistent and for no $\varphi\left(x_{0}\right) \in L$ both $T \cup p \cup\left\{\varphi\left(x_{0}\right)\right\}$ and $T \cup p \cup\left\{\neg \varphi\left(x_{0}\right)\right\}$ are consistent.

An element $b \in|M|$ realizes $p$ if $\varphi\left(x_{0}\right) \in p$ implies $M \vDash \varphi[b]$ ( $\vDash$-satisfaction sign), and $M$ realizes $p$ if some $a \in|M|$ realizes it. A complete theory in $L$ is a maximal consistent set of sentences of $L$. For every permutation $\theta$ of $P$, model $M$, and sublanguage $L$ of $L(M)$ we define an Ehrenfeucht game $E G(M, L, \theta)$ between player I and II with $\omega$ moves as follows: in the $n$th move first player I chooses $i \in\{0,1\}$ and $a_{n}^{i} \in|M|$ and secondly player II chooses $a_{n}^{1-i} \in|M|$. Player II wins if the extension $\theta^{*}$ of $\theta$ defined by $\theta^{*}\left(a_{n}^{0}\right)=a_{n}^{1}$ p eserves all atomic formulas of $L$. That is if $R\left(x_{1}, \cdots, x_{n}\right)$ is an atomic tormula in $L, \theta^{*}\left(b_{i}\right)$ is defined then $M \vDash R\left[b_{1}, \cdots, b_{n}\right]$ iff $M \vDash R\left[\theta^{*}\left(b_{1}\right), \cdots, \theta^{*}\left(b_{n}\right)\right]$. 
REMARK. So if I chooses $a_{n}^{i} \in P$, II should choose $a_{n}^{1}=\theta\left(a_{n}^{0}\right)$.

Define $\Gamma\left(n_{0}\right)=\left\{\theta: \theta\right.$ a permutation of $P, n<n_{0} \Rightarrow \theta\left(c_{n}\right)=c_{n}$ and only for finitely many $\left.n \theta\left(c_{n}\right) \neq c_{n}\right\}$.

$M \mid L$ is the reduct of $M$ to the language $L \subseteq L(M)$, that is $M \mid L$ is $M$ without the relations $R^{M}, R \in L(M), R \notin L$, and constants $c_{n} \in L(M), c_{n} \notin L$.

THEOREM 2. For every ordinal $\alpha<\omega_{1}$ there is a countable firstorder language $L_{\alpha}$ a complete theory $T_{\alpha}$ in $L$, such that

(i ) $p=\left\{P\left(x_{0}\right)\right\} \cup\left\{x_{0} \neq c_{n}: n<\omega\right\}$ is a complete type for $T_{\alpha}$.

(ii) $T_{\alpha}$ has a model of cardinality $\beth_{\alpha}$ omitting $p$.

(iii) $T_{\alpha}$ has no model of cardinality $>\beth_{\alpha}$ omitting $p$.

REMARK. Clearly Theorem 2 implies Theorem 1.

Proof. We shall define by induction on $\alpha<\omega_{1}$ models $M_{\alpha}$ such that

(1) $\left\|M_{\alpha}\right\|$, the cardinality of $\left|M_{\alpha}\right|$, is, $\beth_{\alpha}$, and of course $P=P\left(M_{\alpha}\right)=\left\{c_{n}: n<\omega\right\}$ and except for the $c_{n}$ 's $L\left(M_{\alpha}\right)$ has only predicates.

(2) There is no model elementarily equivalent to $M_{\alpha}$ of cardinality $>\beth_{\alpha}$ which omits $p$.

(3) If $(\exists \beta)(\alpha=\beta+2)$ then $Q_{\alpha} \in L\left(M_{\alpha}\right)$ and $\left|Q_{\alpha}\left(M_{\alpha}\right)\right|=\beth_{\alpha}$

(4) For every finite sublanguage $L$ of $L\left(M_{\alpha}\right)$ there is $n_{L}=$ $n(L)<\omega$, such that for every permutation $\theta \in \Gamma\left(n_{L}\right)$ player II has a winning strategy in $E G\left(M_{\alpha}, L, \theta\right)$.

(5) In (4) if $(\exists \beta)(\alpha=\beta+2)$ then in the winning strategy of II, if I chooses $a_{n}^{i} \in Q_{\alpha}\left(M_{\alpha}\right)$ then II chooses $a_{n}^{1-i}=a_{n}^{i}$.

The induction will go as follows. First we define $M_{0}, M_{1}$, and $M_{2}$; later we define $M_{\alpha+1}$ by $M_{\alpha}$ when $(\exists \beta)(\alpha=\beta+2)$; last for limit ordinal $\delta$ we define $M_{\delta}, M_{\delta+1}, M_{\delta+2}$ by $M_{\alpha} \alpha<\delta$.

But before defining the $M_{\alpha}$ 's, let us show how this will finish the proof. We choose $L_{\alpha}=L\left(M_{\alpha}\right) . \quad T_{\alpha}$ is the set of sentences of $L_{\alpha}$ that $M_{\alpha}$ satisfies. Clearly (ii), (iii) are satisfied. To prove (i) let $\varphi\left(x_{0}\right) \in L_{\alpha}$, so for some finite sublanguage $L$ of $L_{\alpha} \varphi\left(x_{0}\right) \in L$. By possibly interchanging $\varphi\left(x_{0}\right)$ and $\neg \varphi\left(x_{0}\right)$ we can assume $M_{\alpha} \vDash \varphi\left[c_{n(L)}\right]$. For $k \geqq n(L)$ let $\theta_{k}$ be the permutation of $P$ interchanging $c_{n(L)} c_{k}$, and leaving the other elements fixed.

Clearly $\theta \in \Gamma\left(n_{L}\right)$, hence player II has a winning strategy in $E G\left(M_{\alpha}, L, \theta\right)$. By Ehrenfeucht [1] this implies $c_{n(L)}$ and $c_{k}=\theta\left(c_{n(L)}\right)$ satisfy the same formulas of $L$. Hence $M_{\alpha} \vDash \varphi\left[c_{n(L)}\right] \equiv \varphi\left[c_{k}\right]$, hence $M_{\alpha} \vDash \varphi\left[c_{k}\right] . \quad$ As this holds for any $k \geqq n(L) M_{\alpha} \vDash(\forall x)\left[P(x) \wedge \Lambda_{i<n(L)} x \neq\right.$ $\left.c_{i} \rightarrow \varphi(x)\right]$. Hence $T_{\alpha} \cup p \cup\left\{\neg \varphi\left(x_{0}\right)\right\}$ is inconsistent. So $p$ is complete 
(for $T_{\alpha}, L_{\alpha}$ ) and we finish.

So let us define

Case I. $\alpha=0,1,2$

(A) Let us define $M_{0}$ :

$\left|M_{0}\right|=P$, and its only predicate is $P$ (and of course the individual constants $c_{n}$, which we will not mention in later cases). Clearly (1), (2) are immediate. (3) and (5) are satisfied vacuously. As for (4), let $n_{L}=\max \left\{n+1: c_{n} \in L\right\}$. Clearly $\theta$ is an automorphism of $M_{0} \mid L$ (the reduct of $M_{0}$ to $\left.L\right)$.

So player II will play by the automorphism: if I chooses $a_{n}^{0}$, II will choose $a_{n}^{1}=\theta\left(a_{n}^{0}\right)$, and if I chooses $a_{n}^{1}$, II will choose $a_{n}^{0}=\theta^{-1}\left(a_{n}^{1}\right)$.

(B) $\left|M_{1}\right|=\left|M_{0}\right| \cup P_{1}\left(M_{1}\right)$, where $P_{1}\left(M_{1}\right)=\mathscr{P}\left(\left|M_{0}\right|\right)$, where $\mathscr{P}(A)=$ the power set of $A=\{B: B \subseteq A\}$.

The predicates of $M_{1}$ are those of $M_{0}, P_{1}$ and $\varepsilon_{1}$

$$
\varepsilon_{1}\left(M_{1}\right)=\left\{\langle c, A\rangle: c \in\left|M_{0}\right|, A \in P_{1}, c \in A\right\} .
$$

As in (A) it is clear that $M_{1}$ satisfies the induction conditions, as if $\theta \in \Gamma\left(n_{L}\right) L \cong L\left(M_{1}\right), L$ finite, then $\theta$ can be extended to an automorphism of $M_{1}$ by

$$
\theta(A) \stackrel{\text { def }}{=}\{\theta(c): c \in A\} .
$$

(C) Let us define an equivalence relation $E_{1}$ on $P_{1}\left(M_{1}\right): A E_{1} B$ iff for some $\theta \in \Gamma(0) A=\theta(B)[=\{\theta(c): c \in B\}]$.

This is an equivalence relation, as $\Gamma(0)$ is a group of permutations, and as $|\Gamma(0)|=\boldsymbol{\aleph}_{0}$, each equivalence class is countable. Define

$$
\begin{aligned}
\left|M_{2}\right| & =\left|M_{1}\right| \cup Q_{2}\left(M_{2}\right) \\
Q_{2}\left(M_{2}\right) & =\left\{S: S \subseteq P_{1}\left(M_{1}\right), A, B \in P_{1}, A E_{1} B \Rightarrow A \in S \longleftrightarrow B \in S\right\} \\
\varepsilon_{2}\left(M_{2}\right) & =\left\{\langle A, S\rangle: A \in P_{1}, S \in Q_{2}, A \in S\right\} .
\end{aligned}
$$

The relations of $M_{2}$ will be the relations of $M_{1}$, and $Q_{2}, \varepsilon_{2}$. By the definition of $Q_{2}$, each $\theta \in \Gamma\left(n_{L}\right)$ [ $L$ a finite sublanguage of $L\left(M_{2}\right)$ ] can be extended to an automorphism $\theta^{*}$ of $M_{2} \mid L$, which is the identity over $Q_{2}$. As before (1), (2), (4) hold, and as $\theta^{*}$ is the identity over $Q_{2}$, also (5) holds. As for (3) each $E_{1}$-equivalence class is countable, and $\left|P_{1}\left(M_{1}\right)\right|=2^{|P|}=2^{\aleph_{0}}$, the number of $E_{1}$-equivalence classes is $\beth_{1}$, so $\left|Q_{2}\right|=2^{\beth_{1}}=\beth_{2}$.

Case II. We define $M_{\alpha+1}$, where $M_{\alpha}$ is defined, $(\exists \beta)(\alpha=\beta+2)$. Let

$$
\left|M_{\alpha+1}\right|=\left|M_{\alpha}\right| \cup \mathscr{P}\left(Q_{\alpha}\left(M_{\alpha}\right)\right)
$$


The relations of $M_{\alpha+1}$ will be those of $M_{\alpha}$ and in addition $Q_{\alpha+1}\left(M_{\alpha+1}\right)=\mathscr{P}\left(Q_{\alpha}\left(M_{\alpha}\right)\right)$

$$
\varepsilon_{\alpha+1}\left(M_{\alpha+1}\right)=\left\{\langle a, A\rangle: a \in Q_{\alpha}\left(M_{\alpha}\right), A \in Q_{\alpha+1}\left(M_{\alpha+1}\right), a \in A\right\} .
$$

Clearly Conditions (1), (2), (3) are satisfied. As for (4), (5) the winning strategy of player II in $E G\left(M_{\alpha+1}, L, \theta\right)\left[\theta \in \Gamma\left(n_{L}\right)\right]$ will be as follows: when I chooses elements in $\left|M_{\alpha}\right|$ he will pretend all the game is in $\left|M_{\alpha}\right|$ and play accordingly; and if player I chooses $a_{n}^{i} \in Q_{\alpha+1}\left(M_{\alpha+1}\right)$, then player II will choose $a_{n}^{1-i}=a_{n}^{i}$. As $M_{\alpha}$ satisfies (5) this is a winning strategy, and trivially it satisfies (5).

Case III. $\delta$ a limit ordinal, $M_{\alpha}$ is defined for $\alpha<\delta$; and we shall define $M_{\delta}, M_{\delta+1}, M_{\delta+2}$.

PART A. By changing, when necessary, names of elements and relations, we can assume that for $\alpha<\beta<\delta$,

$$
\left|M_{\alpha}\right| \cap\left|M_{\beta}\right|=P \text {, and } L\left(M_{\alpha}\right) \cap L\left(M_{\beta}\right)=\left\{P, c_{n}: n<\omega\right\},
$$

but that if $(\exists \beta)(\alpha=\beta+2)$ then still $Q_{\alpha} \in L\left(M_{\alpha}\right)$. Choose an increasing sequence of ordinals $\alpha_{n} n<\omega, \delta=\bigcup_{n<\omega} \alpha_{n}$ and $(\exists \beta)\left(\alpha_{n}=\beta+2\right)$. Define $M_{o}$ as follows

$$
\left|M_{\delta}\right|=\bigcup_{n<\omega} M_{\alpha_{n}} \text {. }
$$

The relations of $M_{\delta}$ will be those of $M_{\alpha_{n}}$ for each $n<\omega$ and $R_{\delta}^{M o}$

$$
R_{\tilde{\delta}}^{M \grave{o}}=\left\{\langle c, a\rangle: c=c_{n} \in P, a \in\left(M_{\alpha_{n}}-P\right)\right\} .
$$

It is easy to check that Conditions (1), (2) are satisfied. Conditions (3) and (5) are vacuous. So let us prove Condition (4) holds. Let $L$ be a finite sublanguage of $L\left(M_{o}\right)$; then $L \subseteq \bigcup_{j<n_{0}} L_{j} \cup\{R\}$, where $L_{j}=L \cap L\left(M_{\alpha_{j}}\right)$ is a finite sublanguage of $L\left(M_{\alpha_{j}}\right)$. Define $n_{L}=$ $\max \left[\left\{n_{L_{j}}: j<n_{0}\right\} \cup\left\{n_{0}\right\}\right]$. Let $\theta \in \Gamma\left(n_{L}\right)$. We shall describe now the winning strategy of player II in $E G\left(M_{\delta}, L, \theta\right)$. When player I will choose $i \in\{0,1\}, a_{n}^{i} \in M_{\alpha_{j}}, j<n_{0}$, player II will pretend all the game is in the model $M_{\alpha_{j}}$, and so play his winning strategy for $E G\left(M_{\alpha_{j}}, L \cap\right.$ $\left.L\left(M_{\alpha_{j}}\right), \theta\right)$. If piayer I chooses $i \in\{0,1\}, a_{n}^{i} \in M_{\alpha_{j}} j \geqq n_{0}$ then player II will choose $a_{n}^{1-i} \in M_{\alpha_{k}}$ [where $i=0 \Rightarrow k=\theta(j), i=1 \Rightarrow j=\theta(k)$ ] such that for any $m<n a_{m}^{i}=a_{n}^{i} \Leftrightarrow a_{m}^{1-i}=a_{n}^{1-i}$.

Note that for $j \geqq n_{0}$, in $M_{\delta} \mid L$, every permutation of elements of $M_{\alpha_{j}}$ is an automorphism, as the only relation an $a \in\left|M_{\alpha_{j}}\right|$ satisfies is $R_{\hat{o}}\left[c_{j}, a\right]$.

PART B. Here we define $M_{\hat{o}+1}$ • Let $A^{*}=\bigcup_{n<\omega} Q_{\alpha_{n}}\left(M_{\alpha_{n}}\right)$, and $\left|M_{\delta+1}\right|=\left|M_{\delta}\right| \cup \mathscr{P}\left(A^{*}\right)$. 
The relations of $M_{\delta+1}$ will be those of $M_{\delta}$, and in addition

$$
\begin{aligned}
P_{\delta}\left(M_{\delta+1}\right) & =\left|M_{\delta}\right|, P_{\delta+1}\left(M_{\delta+1}\right)=\mathscr{P}\left(A^{*}\right) \\
\varepsilon_{\delta+1}\left(M_{\delta+1}\right) & =\left\{\langle b, B\rangle: b \in A^{*}, B \in \mathscr{P}\left(A^{*}\right), b \in B\right\} .
\end{aligned}
$$

It is easy to see that Conditions (1), (2) are satisfied, and (3), (5) are vacuous. So let us prove $(4)$ - let $L$ be a finite sublanguage of $L\left(M_{\tilde{\delta}+1}\right)$. So

$$
L \subseteq \bigcup_{i<n_{0}} L_{i} \cup\left\{R_{\delta}, P_{\delta}, P_{\delta+1}, \varepsilon_{\delta+1}\right\}, L_{i}=L \cap L\left(M_{\alpha_{i}}\right)
$$

Define again

$$
n_{L}=\max \left[\left\{n_{L_{j}}: j<n_{0}\right\} \cup\left\{n_{0}\right\}\right] .
$$

Let $\theta \in \Gamma\left(n_{L}\right)$ and we should describe player II's winning strategy in $E G\left(M_{\delta+1}, L, \theta\right)$. When player I chooses an element in $M_{\alpha_{j}} j<n_{0}$, player II will ignore all elements chosen outside $M_{\alpha_{j}}$, and play by his winning strategy in $E G\left(M_{\alpha_{j}}, L_{j}, \theta\right)$. In the other cases player II will play so that the following conditions are satisfied for every $n$

$P(1) \quad a_{n}^{0} \in P_{\delta+1}\left(M_{\delta+1}\right) \Leftrightarrow a_{n}^{1} \in P_{\delta+1}\left(M_{\delta+1}\right)$

$P(2)$ if $c_{j}=\theta\left(c_{k}\right)$, then $a_{n}^{0} \in\left|M_{\alpha_{k}}\right| \Leftrightarrow a_{n}^{1} \in\left|M_{\alpha_{j}}\right|$

$P(3)$ if $m<n$ then $a_{m}^{0}=a_{n}^{0} \Leftrightarrow a_{m}^{1}=a_{n}^{1}$

$P(4)$ if $m, l \leqq n$ and $a_{m}^{0} \in A^{*}, a_{l}^{0} \in P_{\delta+1}$ then $a_{m}^{0} \in a_{l}^{0} \Leftrightarrow a_{m}^{1} \in a_{l}^{1}$

$P(5)$ if $a_{m}^{0} \in P_{\delta+1}, l<\omega, c_{l}=\theta\left(c_{l}\right)$ then $a_{m}^{0} \cap Q_{\alpha_{l}}\left(M_{\alpha_{l}}\right)=a_{m}^{1} \cap Q_{\alpha_{l}}\left(M_{\alpha_{l}}\right)$

$P(6)$ if $c_{j}=\theta\left(c_{k}\right) j \neq k<\omega$, then $\left\langle a_{m}^{0}: m \leqq n, a_{m}^{0} \in P_{\delta+1}\right\rangle$ and $\left\langle a_{m}^{1}: m \leqq n, a_{m}^{1} \in P_{\delta+1}\right\rangle$ genarate corresponding finite Boolean algebras of subsets of $Q_{\alpha_{k}}\left(M_{\alpha_{k}}\right)$ and $Q_{\alpha_{j}}\left(M_{\alpha_{j}}\right)$ correspondingly; then the corresponding atoms in those algebras are both infinite, or have the same power.

It is easy to see that this can by done, and it is a winning strategy.

PART C. Here we define $M_{\delta+2}$.

Define equivalence relations $E_{\delta+1}, E_{j+1}^{n}$ on $P_{\delta+1}\left(M_{\delta+1}\right)$ : if $A, B \in$ $P_{\delta+1}\left(M_{\tilde{\delta}+1}\right)$, then $A, B \subseteq A^{*}=\bigcup_{n<\omega} Q_{\alpha_{n}}\left(M_{\alpha_{n}}\right)$; define $A E_{\tilde{\delta}+1}^{n} B$ iff $A \cap$ $\left[\bigcup_{\omega>m>n} Q_{\alpha_{m}}\left(M_{\alpha_{m}}\right)\right]=B \cap\left[\bigcup_{\omega>m>n} Q_{\alpha_{m}}\left(M_{\alpha_{m}}\right)\right] ; A E_{\delta+1} B$ iff for some $n$ $A E_{\delta+1}^{n} B$.

Clearly each $E_{\delta+1}^{n}$ is an equivalence relation, $E_{\delta+1}^{n}$ refines $E_{\delta+1}^{n+1}$, hence $E_{\hat{o}+1}$ is an equivalence relation.

It is clear that

$$
\left|P_{\delta+1}\left(M_{\delta+1}\right)\right|=\beth_{\tilde{\delta}+1}
$$

but for every $n<\omega, A \in P_{\delta+1}\left(M_{\tilde{\delta}+1}\right)$ 


$$
\begin{gathered}
\left|\left\{B: B \in P_{\delta+1}\left(M_{\delta+1}\right), B E_{\delta+1}^{n} A\right\}\right| \leqq\left|\mathscr{P}\left(\bigcup_{m \leqq n} Q_{\alpha_{m}}\left(M_{\alpha_{m}}\right)\right)\right| \\
=2^{\beth_{\alpha_{n}}}=\beth_{\alpha_{n}+1} \leqq \beth_{\delta}
\end{gathered}
$$

hence

$$
\left|\left\{B: B \in P_{\delta+1}\left(M_{\delta+1}\right), B E_{\delta+1} A\right\}\right| \leqq \sum_{n<\omega} \beth_{\delta}=\beth_{\delta} .
$$

So each $E_{\delta+1}$ - equivalence class has cardinality $\leqq \beth_{\delta}$, hence there are $\beth_{\hat{\delta}+1} E_{\delta+1}$-equivalence classes.

Define $M_{\hat{o}+2}$ :

$$
\left|M_{\delta+2}\right|=\left|M_{\delta+1}\right| \cup Q_{\delta+2}\left(M_{\delta+2}\right)
$$

where

$Q_{\tilde{\delta}+2}\left(M_{\tilde{\delta}+2}\right)=\left\{S: S \subseteq P_{\delta+1}\left(M_{\tilde{\delta}+1}\right), A, B \in S, A E_{\hat{\delta}+1} B \Longrightarrow A \in S \longleftrightarrow B \in S\right\}$.

Clearly $\left|Q_{\hat{\delta}+2}\left(M_{\tilde{\delta}+2}\right)\right|=\beth_{\hat{o}+2}$.

The relations of $M_{\delta+2}$ will be those of $M_{\delta+1}$, and $Q_{\delta+2}$, and

$$
\varepsilon_{\tilde{\delta}+2}\left(M_{\tilde{\delta}+2}\right)=\left\{\langle A, S\rangle: A \in P_{\tilde{\delta}+1}\left(M_{\tilde{\delta}+1}\right), S \in P_{\tilde{\delta}+2}\left(M_{\delta+2}\right), A \in S\right\} .
$$

It is easy to prove all conditions are satisfied as in Case II, if we notice that by Condition $P(5)$ if for any instance of any game $E G\left(M_{\delta+1}, L, \theta\right)\left[\theta \in \Gamma\left(n_{L}\right)\right]$ in which player II plays his strategy, if $a_{n}^{i}, a_{n}^{1-i}$ are chosen for some $n$ and they belong to $P_{\delta+1}\left(M_{\delta+1}\right)$ then they are $E_{\delta+1}$-equivalent (as $\left\{n: \theta\left(c_{n}\right) \neq n\right\}$ is finite).

\section{REFERENCES}

1. A. Ehrenfeucht, Application of games to completeness problem for formalized theories, Fund. Math., 49 (1961), 129-141.

2. J. Malitz, On the Hanf Number of Complete $L_{\omega_{1}, \omega}$ Sentences; The syntax and semantics of infinitary languages, edited by J. Barwise, Lecture notes in Mathematics 72, Springer, Berlin.

3. M. Morley, Omitting Classes of Elements; The Theory of Models, Proc. of the 1962 Symp. in Berkeley, Editors J. Addison, L. Henkin, and A. Tarski, North-Holland Publ. Co., Amsterdam, 1965, 265-273.

Received May 18, 1972.

The Hebrew UnIVERSITY 


\section{PACIFIC JOURNAL OF MATHEMATICS}

\section{EDITORS}

RICHARD ARENS (Managing Editor) University of California

Los Angeles, California 90024

R. A. BeaUmont

University of Washington Seattle, Washington 98105
J. DUGUNDJI*

Department of Mathematics University of Southern California Los Angeles, California 90007

D. Gilbarg and J. Milgram Stanford University

Stanford, California 94305

\section{ASSOCIATE EDITORS}

E. F. BECKENBACH

B. H. NeUmanN

F. WOLF

K. YoSHIDA

\section{SUPPORTING INSTITUTIONS}

\section{UNIVERSITY OF BRITISH COLUMBIA CALIFORNIA INSTITUTE OF TECHNOLOGY UNIVERSITY OF CA.LIFORNIA MONTANA STATE UNIVERSITY UNIVERSITY OF NEVADA NEW MEXICO STATE UNIVERSITY OREGON STATE UNIVERSITY UNIVERSITY OF OREGON OSAKA UNIVERSITY}

UNIVERSITY OF SOUTHERN CALIFORNIA STANFORD UNIVERSITY UNIVERSITY OF TOKYO UNIVERSITY OF UTAH WASHINGTON STATE UNIVERSITY UNIVERSITY OF WASHINGTON AMERICAN MATHEMATICAL SOCIETY NAVAL WEAPONS CENTER

* C. R. DePrima California Institute of Technology, Pasadena, CA 91109, will replace J. Dugundji until August 1974. 


\section{Pacific Journal of Mathematics}

\section{Vol. 50, No. $1 \quad$ September, 1974}

Gail Atneosen, Sierpinski curves in finite 2-complexes.............. 1

Bruce Alan Barnes, Representations of $B^{*}$-algebras on Banach spaces .... 7

George Benke, On the hypergroup structure of central $\Lambda(p)$ sets ....... 19

Carlos R. Borges, Absolute extensor spaces: a correction and an

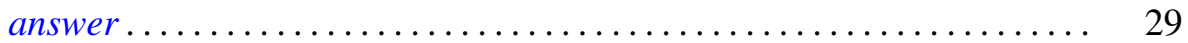

Tim G. Brook, Local limits and tripleability .................. 31

Philip Throop Church and James Timourian, Real analytic open maps .... 37

Timothy V. Fossum, The center of a simple algebra ............... 43

Richard Freiman, Homeomorphisms of long circles without periodic

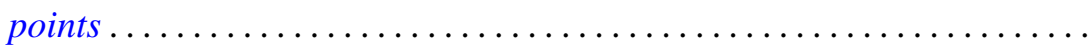

B. E. Fullbright, Intersectional properties of certain families of compact

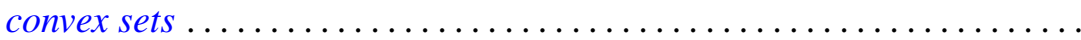

Harvey Charles Greenwald, Lipschitz spaces on the surface of the unit

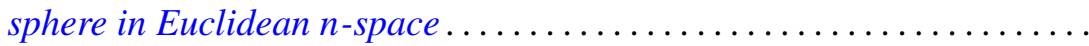

Herbert Paul Halpern, Open projections and Borel structures for

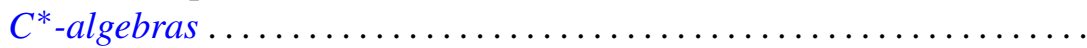

Frederic Timothy Howard, The numer of multinomial coefficients divisible

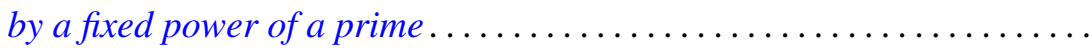

Lawrence Stanislaus Husch, Jr. and Ping-Fun Lam, Homeomorphisms of manifolds with zero-dimensional sets of nonwandering points........ 109

Joseph Edmund Kist, Two characterizations of commutative Baer rings ...

Lynn McLinden, An extension of Fenchel's duality theorem to saddle functions and dual minimax problems ................

Leo Sario and Cecilia Wang, Counterexamples in the biharmonic classification of Riemannian 2-manifolds...

Saharon Shelah, The Hanf number of omitting complete types ...

Richard Staum, The algebra of bounded continuous functions into a

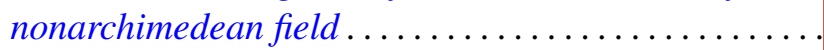

James DeWitt Stein, Some aspects of automatic continuity ..

Tommy Kay Teague, On the Engel margin

John Griggs Thompson, Nonsolvable finite groups all of whose local subgroups are solvable, $V \ldots \ldots \ldots \ldots \ldots \ldots \ldots \ldots$

Kung-Wei Yang, Isomorphisms of group extensions 